\title{
A note on generalized flag structures
}

\author{
by Tomasz Rybicki (Rzeszów)
}

\begin{abstract}
Generalized flag structures occur naturally in modern geometry. By extending Stefan's well-known statement on generalized foliations we show that such structures admit distinguished charts. Several examples are included.
\end{abstract}

1. Introduction. It has been first established by P. Stefan in $[13,14]$ that the orbits of any "isotopically connected" set of local $C^{r}$-diffeomorphisms, $1 \leq r \leq \omega$, fit together to form a generalized foliation. The career of this statement in geometry is justified by the fact that a nontransitive geometric structure usually induces a foliation with singularities. Further facts concerning generalized foliations and the integrability of distributions can be found in $[1,2,15,17]$. However, it seems that surprisingly little is known on this subject compared with the theory of regular foliations.

In this note we give some introductory remarks on generalized flag structures. This notion describes a somewhat more complicated situation which arises for instance in multisymplectic geometry, Riemannian foliations, Jacobi structures, Hamiltonian actions on Poisson manifolds (cf. Section 3).

Given generalized foliations $\mathcal{F}_{i}(i=1,2)$ on a manifold $M$ we write $\mathcal{F}_{1} \prec \mathcal{F}_{2}$ and say that $\mathcal{F}_{1}$ is a subfoliation of $\mathcal{F}_{2}$ if every leaf of $\mathcal{F}_{1}$ is contained in a leaf of $\mathcal{F}_{2}$. By a generalized flag structure on $M$ we mean any finite sequence $\mathcal{F}_{1} \prec \ldots \prec \mathcal{F}_{k}$ of foliations of $M$. Throughout we shall drop the term "generalized" in the above notions.

Our purpose is to formulate the notion of a distinguished chart for a flag structure. We show that any flag structure admits distinguished charts. This fact is well known and trivial for regular flag structures (see, e.g., [18]) in contrast to the case of (regular) almost-product structures where one needs more than merely the integrability of each distribution to obtain the

1991 Mathematics Subject Classification: Primary 57R30.

Key words and phrases: generalized foliation, subfoliation, flag structure, distinguished chart.

Partially supported by KBN 2 P03A 02410. 
integrability of the whole structure. One of the consequences of our result is that the pseudo- $n$-transitivity can be formulated in a more general context (Section 4).

2. The existence of distinguished charts. Let us recall some concepts from [13] and [14]. A foliation of class $C^{r}, 1 \leq r \leq \omega$, is a partition $\mathcal{F}$ of $M$ into weakly imbedded submanifolds (see below), called leaves, such that the following condition holds. If $x$ belongs to a $k$-dimensional leaf, there exists an (inverse) chart $(U, \phi)$ with $\phi(0)=x$ and $U=V \times W$, where $V$ is an open ball in $\mathbb{R}^{k}$ and $W$ is an open ball in $\mathbb{R}^{n-k}$, such that if $L \in \mathcal{F}$ then

$$
\phi(U) \cap L=\phi(V \times l), \quad \text { for } l=\{w \in W: \phi(0, w) \in L\} .
$$

A subset $L$ of a $C^{r}$-manifold $M$ endowed with a $C^{r}$-differentiable structure $\sigma$ which makes it an immersed submanifold is weakly imbedded if for any locally connected topological space $N$ and a continuous map $f: N \rightarrow M$ satisfying $f(N) \subset L$, the map $f: N \rightarrow L$ is continuous as well. It follows that such a differentiable structure $\sigma$ is necessarily unique.

A smooth mapping $\phi$ of an open subset of $\mathbb{R} \times M$ into $M$ is said to be a $C^{r}$-arrow if $(1) \phi(t, \cdot)=\phi_{t}$ is a local $C^{r}$-diffeomorphism for each $t$, possibly with empty domain, (2) $\phi_{0}=$ id on its domain, and $(3) \operatorname{dom}\left(\phi_{t}\right) \subset \operatorname{dom}\left(\phi_{s}\right)$ whenever $0 \leq s<t$.

Given an arbitrary set $A$ of arrows let $A^{*}$ be the totality of local diffeomorphisms $\psi$ such that $\psi=\phi(t, \cdot)$ for some $\phi \in A, t \in \mathbb{R}$. Next, $A_{*}$ stands for the set consisting of all local diffeomorphisms which are finite compositions of elements from $A^{*}$ or $\left(A^{*}\right)^{-1}=\left\{\psi^{-1}: \psi \in A^{*}\right\}$ and of the identity. Then the orbits of $A_{*}$ are called accessible sets of $A$.

For $x \in M$ we let $A^{0}(x), A(x)$, and $\bar{A}(x)$ be the vector subspaces of $T_{x} M$ spanned by

$$
\{\dot{\phi}(0, x): \phi \in A\}, \quad\left\{\dot{\phi}(t, y): \phi \in A, \phi_{t}(y)=x\right\},
$$

and

$$
\left\{d_{y} \psi(v): \psi \in A_{*}, \psi(y)=x, v \in A(y)\right\},
$$

respectively. Clearly $A^{0}(x) \subset A(x) \subset \bar{A}(x)$.

THEOREM 1 [13]. Let $A$ be an arbitrary set of $C^{r}$-arrows on $M$. Then:

(i) Every accessible set of $A$ admits a (unique) $C^{r}$-differentiable structure of a connected weakly imbedded submanifold of $M$.

(ii) The collection of accessible sets defines a foliation $\mathcal{F}=\mathcal{F}(A)$.

(iii) $\{\bar{A}(x)\}_{x \in M}$ is the tangent distribution of $\mathcal{F}(A)$.

Any diffeomorphism group $G(M) \subset \operatorname{Diff}^{r}(M)$ defines uniquely a set of arrows. Namely, by a $C^{r}$-smooth path (or isotopy) in $G(M)$ we mean any family $\left\{f_{t}\right\}_{t \in \mathbb{R}}$ with $f_{t} \in G(M)$ such that the map $(t, x) \mapsto f_{t}(x)$ is smooth. 
Next, $G(M)_{0}$ denotes the subgroup of all $f \in G(M)$ such that there is a smooth path $\left\{f_{t}\right\}_{t \in \mathbb{R}}$ with $f_{t}=$ id for $t \leq 0$ and $f_{t}=f$ for $t \geq 1$. The totality of $f_{t}$ as above constitutes a set of arrows. This set determines uniquely a foliation $\mathcal{F}(G)$ which coincides with the orbits of $G(M)_{0}$.

Likewise, any set of local vector fields defines a foliation as any flow is an arrow.

A set of arrows $A$ is said to be homogeneous if $A(x)=\bar{A}(x)$, for all $x \in M$. Next, $A$ is symmetric if $\phi \in A^{*}$ implies that $\phi^{-1}$ is a composition of elements of $A^{*}$.

LEMmA. To each set of arrows $A$ one can assign a homogeneous set of arrows $\mathcal{A}$ such that $\mathcal{F}(\mathcal{A})=\mathcal{F}(A)$ and $\mathcal{A}^{0}(x)=\mathcal{A}(x)$ for all $x \in M$. Moreover, if $A_{1} \subset A_{2}$ then $\mathcal{A}_{1} \subset \mathcal{A}_{2}$, where $\mathcal{A}_{i}$ is assigned to $A_{i}$.

We reproduce the proof from [13] for the sake of completeness.

First we enlarge $A$ by setting

$$
\widetilde{A}=\left\{\phi_{t+s} \circ\left(\phi_{s}\right)^{-1}, \phi_{s-t} \circ\left(\phi_{s}\right)^{-1}: \phi \in A, s \in \mathbb{R}\right\} .
$$

It is visible that $\widetilde{A}_{*}=A_{*}$ and $\mathcal{F}(\widetilde{A})=\mathcal{F}(A)$. Furthermore, $\widetilde{A}$ is symmetric, $\widetilde{A}^{0}(x)=\widetilde{A}(x)=A(x)$, and $\widetilde{A}$ is homogeneous whenever so is $A$.

Next we put

$$
\widehat{A}=\left\{\chi=\psi \circ \phi_{t} \circ \psi^{-1}: \operatorname{dom} \chi=(-\varepsilon, \varepsilon) \times V, V \text { open, } \phi \in \widetilde{A}, \psi \in A_{*}\right\} .
$$

Then by a straightforward inspection $\widehat{A}(x)=\bar{A}(x)$. Since $\mathcal{F}(\widehat{A})=\mathcal{F}(A)$ it follows by Theorem 1 that $\widehat{A}$ is homogeneous.

Therefore $\mathcal{A}=\widetilde{\widehat{A}}$ satisfies the claim.

Let $\mathcal{F}_{1} \prec \ldots \prec \mathcal{F}_{k}$ be a flag structure on $M$ and let $x \in M$. If $x \in$ $L_{i} \in \mathcal{F}_{i}$ we write $p_{i}(x)=\operatorname{dim} L_{i}, \bar{p}_{i}(x)=p_{i}(x)-p_{i-1}(x)(i=2, \ldots, k)$ and $q_{i}(x)=m-p_{i}(x)$.

Definition. A chart $(U, \phi)$ of $M$ with $\phi(0)=x$ is called a distinguished chart at $x$ with respect to $\mathcal{F}_{1} \prec \ldots \prec \mathcal{F}_{k}$ if $U=V_{1} \times \ldots \times V_{k} \times W$ with $V_{1} \subset \mathbb{R}^{p_{1}(x)}, V_{i} \subset \mathbb{R}^{\bar{p}_{i}(x)}(i \geq 2)$ and $W \subset \mathbb{R}^{q_{k}(x)}$ open balls such that for any $L_{i} \in \mathcal{F}_{i}$ we have

$$
\phi(U) \cap L_{i}=\phi\left(V_{1} \times \ldots \times V_{i} \times l_{i}\right),
$$

where

$$
l_{i}=\left\{w \in V_{i+1} \times \ldots \times V_{k} \times W: \phi(0, w) \in L_{i}\right\}
$$

for $i=1, \ldots, k$.

Observe that actually the above $\phi$ is an inverse chart; following [13] we call it a chart for simplicity. Notice as well that in the above definition one need not assume that $\mathcal{F}_{i}$ is a foliation but only that it is a partition 
into weakly imbedded submanifolds; that $\mathcal{F}_{i}$ is a foliation follows then by definition.

THEOREM 2. Let $A_{1} \subset \ldots \subset A_{k}$ be an increasing sequence of sets of arrows of $M$. Then $\mathcal{F}\left(A_{1}\right) \prec \ldots \prec \mathcal{F}\left(A_{k}\right)$ admits a distinguished chart at any $x \in M$. Specifically, so does any flag structure on $M$.

Proof. Fix $x \in M$ and let $p_{i}=p_{i}(x), \bar{p}_{i}=\bar{p}_{i}(x), q_{i}=q_{i}(x)$. We have $\bar{A}_{i}(x)=T_{x} \mathcal{F}_{i}, i=1, \ldots, k$. In view of Lemma each $A_{i}$ can be replaced by $\mathcal{A}_{i}$ such that $\mathcal{A}_{i}^{0}(x)=\bar{A}_{i}(x), \mathcal{F}\left(\mathcal{A}_{i}\right)=\mathcal{F}\left(A_{i}\right)$, and $\mathcal{A}_{1} \subset \ldots \subset \mathcal{A}_{k}$.

First we choose $\phi^{j} \in \mathcal{A}_{1}^{*}, j=1, \ldots, p_{1}$, such that $\dot{\phi}^{j}(0, x)$ form a basis of $T_{x} \mathcal{F}_{1}$. Next we extend this basis to a basis of $T_{x} \mathcal{F}_{2}$ by means of $\dot{\phi}^{j}(0, x)$ with $\phi^{j} \in \mathcal{A}_{2}^{*}$ for $j=p_{1}+1, \ldots, p_{2}$, and so on. Thus we obtain a basis of $T_{x} \mathcal{F}_{k}$ of the form $\dot{\phi}^{1}(0, x), \ldots, \dot{\phi}^{p_{k}}(0, x)$ where $\phi^{1}, \ldots, \phi^{p_{i}} \in \mathcal{A}_{i}^{*}$ for $i=1, \ldots, k$.

Now let $Q$ be a $q_{k}$-dimensional submanifold of $M$ with $x \in Q$ and $T_{x} M=$ $T_{x} \mathcal{F}_{k} \oplus T_{x} Q$. Shrinking $Q$ if necessary we may and do assume that

$$
\dot{\phi}^{p_{k-1}+1}(0, z), \ldots, \dot{\phi}^{p_{k}}(0, z)
$$

are linearly independent for any $z \in Q$. By choosing $\varepsilon_{k}>0$ sufficiently small we see that the mapping

$$
\psi_{k}:\left(t_{p_{k-1}+1}, \ldots, t_{p_{k}}, z\right) \in V_{k} \times Q \mapsto \phi_{t_{p_{k-1}+1}}^{p_{k-1}+1} \circ \ldots \circ \phi_{t_{p_{k}}}^{p_{k}}(z) \in M
$$

is an imbedding, where $V_{k}=\left\{t=\left(t_{p_{k-1}+1}, \ldots, t_{p_{k}}\right) \in \mathbb{R}^{\bar{p}_{k}}:|t|<\varepsilon_{k}\right\},|\cdot|$ being the usual norm. Then $T_{x} M=T_{x} \mathcal{F}_{k-1} \oplus T_{x}\left(\operatorname{im} \psi_{k}\right)$. Again, possibly shrinking $Q$ and $V_{k}$ we find that

$$
\dot{\phi}^{p_{k-2}+1}\left(0, z^{\prime}\right), \ldots, \dot{\phi}^{p_{k-1}}\left(0, z^{\prime}\right)
$$

are linerly independent for any $z^{\prime} \in \operatorname{im} \psi_{k}$. Then with some small $\varepsilon_{k-1}>0$ the mapping

$\psi_{k-1}:\left(t_{p_{k-2}+1}, \ldots, t_{p_{k}}, z\right) \in V_{k-1} \times V_{k} \times Q \mapsto \phi_{t_{p_{k-2}+1}}^{p_{k-2}+1} \circ \ldots \circ \phi_{t_{p_{k}}}^{p_{k}}(z) \in M$

is an imbedding, where $V_{k-1}=\left\{t=\left(t_{p_{k-2}+1}, \ldots, t_{p_{k-1}}\right) \in \mathbb{R}^{\bar{p}_{k-1}}:|t|<\right.$ $\left.\varepsilon_{k-1}\right\}$. We have $T_{x} M=T_{x} \mathcal{F}_{k-2} \oplus T_{x}\left(\operatorname{im}\left(\psi_{k-1}\right)\right)$.

By continuing this procedure we obtain a chart of the form

$$
\psi_{1}=\phi:\left(t_{1}, \ldots, t_{p_{k}}, y\right) \in V_{1} \times \ldots \times V_{k} \times W \mapsto \phi_{t_{1}}^{1} \circ \ldots \circ \phi_{t_{p_{k}}}^{p_{k}}(\chi(y)) \in M \text {, }
$$

where $W \subset \mathbb{R}^{q_{k}}$ is an open ball and $\chi: W \rightarrow Q$ with $\chi(0)=x$ is an (inverse) chart.

Let us check that $\phi$ is distinguished, i.e. that $(*)$ is fulfilled. Fix $1 \leq i \leq k$. Let $z \in U$ and $\phi(z)=\phi_{t_{1}}^{1} \circ \ldots \circ \phi_{t_{p_{k}}}^{p_{k}}(\chi(y))=\phi_{t_{1}}^{1} \circ \ldots \circ \phi_{t_{p_{i}}}^{p_{i}}(v)$ for some $y \in W$ and $v \in \operatorname{im}\left(\psi_{k-i+1}\right)$. But $\operatorname{im}\left(\psi_{k-i+1}\right)=\phi\left(0 \times \ldots \times 0 \times V_{i+1} \times \ldots \times V_{k} \times W\right)$. Hence $\phi(z) \in L_{i}$ if and only if $v \in L_{i} \cap \operatorname{im}\left(\psi_{k-i+1}\right)$, i.e. $v=\phi(0, w)$ where $w \in l_{i}$. In fact, $\phi_{t}^{1}, \ldots, \phi_{t}^{p_{i}}$ preserve all the leaves of $\mathcal{F}_{i}$. 
For the second assertion let $\mathcal{F}_{1} \prec \ldots \prec \mathcal{F}_{k}$ be a flag structure. If $A\left(\mathcal{F}_{i}\right)$ is the set of flows of all (local) vector fields tangent to $\mathcal{F}_{i}$ then obviously $\mathcal{F}\left(A\left(\mathcal{F}_{i}\right)\right)=\mathcal{F}_{i}$. The proof follows by the first part.

In view of the above proof we get

Corollary. Let $\mathcal{F}_{1} \prec \ldots \prec \mathcal{F}_{k}$ on $M$ and let $(L, \sigma)$ be a leaf of $\mathcal{F}_{k}$. Then $\mathcal{F}_{1}\left|L \prec \ldots \prec \mathcal{F}_{k-1}\right| L$ and a distinguished chart at $x$ for $L$ is the restriction to $L$ of a distiguished chart at $x$ for $M$.

3. Examples. We give some geometric examples of subfoliations or flag structures which motivate our interest, mainly in disciplines being nowadays intensively developed.

3.1. Let $\mathcal{F}_{i}, i=1,2$, be a foliation of class $C^{\infty}$ and $D_{i}=\left\{T_{x} \mathcal{F}_{i}\right\}$ its tangent distribution. Then $\mathcal{F}_{1} \cap \mathcal{F}_{2} \prec \mathcal{F}_{i}$ if and only if $D=D_{1} \cap D_{2}$ is of class $C^{\infty}$ (i.e. for any $x \in M$ there are vector fields $X_{1}, \ldots, X_{k(x)}$ such that $D_{x}$ is spanned by $\left.X_{1}(x), \ldots, X_{k(x)}(x)\right)$ (cf. [1]).

3.2. Let $G_{i}(M) \subset \operatorname{Diff}^{r}(M)$ be a locally arcwise connected group of diffeomorphisms. If $G_{1}(M) \subset \ldots \subset G_{k}(M)$ we get a flag structure $\mathcal{F}\left(G_{1}\right) \prec$ $\ldots \prec \mathcal{F}\left(G_{k}\right)$.

3.3. Recall that any set of local vector fields $\mathcal{V}(M)$ on $M$ defines uniquely a foliation $\mathcal{F}(\mathcal{V})$. So if $\mathcal{V}_{1} \subset \ldots \subset \mathcal{V}_{k}$ is an increasing sequence of sets of local vector fields one has $\mathcal{F}\left(\mathcal{V}_{1}\right) \prec \ldots \prec \mathcal{F}\left(\mathcal{V}_{k}\right)$. In particular, if $X$ is any vector field tangent to a foliation $\mathcal{F}$ then the orbits of its flow constitute a subfoliation of $\mathcal{F}$.

3.4. On $M=\mathbb{R}^{3}$ we define two foliations:

$\mathcal{F}_{1}=\left\{\right.$ all circles parallel to the $x_{2} x_{3}$-plane with center on the $x_{1}$-axis $\}$, and

$$
\mathcal{F}_{2}=\{\text { all spheres centered at } 0\} .
$$

Then $\mathcal{F}_{1} \prec \mathcal{F}_{2}$. We have three types of points: (i) if $x=0$ then $p_{1}(x)=$ $p_{2}(x)=0$; (ii) if $x$ lies on the $x_{1}$-axis, $x \neq 0$ then $p_{1}(x)=0$ and $p_{2}(x)=2$; (iii) $p_{1}(x)=1$ and $p_{2}(x)=2$ for $x$ off the $x_{1}$-axis.

3.5. Let $(M, \mathcal{F})$ be a regular Riemannian foliation and let $\overline{\mathcal{F}}$ be the set of closures of the leaves of $\mathcal{F}$. Then $\overline{\mathcal{F}}$ is a singular Riemannian foliation (cf. [9]) and obviously $\mathcal{F} \prec \overline{\mathcal{F}}$.

Also orbit-like foliations introduced by P. Molino in [10] have the property that the foliation $\overline{\mathcal{F}}$ by the closures of leaves of $\mathcal{F}$ is again Riemannian, and the relation $\mathcal{F} \prec \overline{\mathcal{F}}$ holds.

3.6. Let $\Lambda_{i}$ be a Poisson structure on $M, i=1, \ldots, k$, and let $\mathcal{F}\left(\Lambda_{i}\right)$ be the corresponding symplectic foliation (cf. [16]). If $\mathcal{F}\left(\Lambda_{1}\right) \prec \ldots \prec \mathcal{F}\left(\Lambda_{k}\right)$ we 
shall say that $\left(\Lambda_{1}, \ldots, \Lambda_{k}\right)$ constitutes a Poisson flag structure. Observe that in contrast to Theorem 2 and despite the existence of the canonical charts for Poisson manifolds (a splitting theorem of Weinstein [16]), a common canonical chart for $\Lambda_{i}$ would exist only in very special cases.

Likewise, a Jacobi flag structure arises when one has a sequence of Jacobi structures $\left(\Lambda_{i}, E_{i}\right)$ such that $\mathcal{F}\left(\Lambda_{1}, E_{1}\right) \prec \ldots \prec \mathcal{F}\left(\Lambda_{k}, E_{k}\right)$, where $\mathcal{F}\left(\Lambda_{i}, E_{i}\right)$ is the characteristic foliation of $\left(\Lambda_{i}, E_{i}\right)$ (see (3.10) below).

3.7 (Reduction of Poisson manifolds, cf. [16]). Let $N$ be a submanifold of a Poisson manifold $(M, \Lambda)$ such that $\mathcal{D}_{N}=(\sharp \operatorname{Ann}(T N)) \cap T N$ is a distribution of constant dimension along $N$. Here $\sharp: \Omega^{1}(M) \rightarrow \mathcal{X}(M)$ is defined by $\sharp(\alpha)(\beta)=\Lambda(\alpha, \beta)$, and Ann denotes the annihilator. Then $\mathcal{D}_{N}$ is differentiable and integrates to a foliation $\mathcal{F}_{N}$ which is called the subcharacteristic foliation of $N$. If $N$ is transversal to $\mathcal{F}(\Lambda)$ then $\mathcal{F}_{N} \prec \mathcal{F}(\Lambda) \cap N$.

3.8. Consider a Hamiltonian action of a compact Lie group $G$ on a Poisson manifold $(M, \Lambda)$ (cf. [11]). Then the orbits of $G$ form a subfoliation $\mathcal{F}_{G}$ of $\mathcal{F}(\Lambda)$. The same is true for canonical manifolds (cf. [6]).

3.9. A homogeneous Poisson structure $(\Lambda, Z)$ on a manifold $M$ is a Poisson structure $\Lambda$ and a vector field $Z$ such that $\mathcal{L}_{Z} \Lambda=-\Lambda$, where $\mathcal{L}$ is the Lie derivative. These structures play a central role in the theory of Jacobi manifolds (see [3]). Let $\mathcal{F}(\Lambda, Z)$ be the foliation generated by $\mathcal{F}(\Lambda)$ and $Z$ (at some points $Z$ is tangent to $\mathcal{F}(\Lambda)$ and at some is not). Then $\mathcal{F}(\Lambda) \prec \mathcal{F}(\Lambda, Z)$. This is still the case of locally homogeneous structures (cf. [3, Prop. 2.16]).

3.10. Recall that a pair $(\Lambda, E)$ is a Jacobi structure on $M$ if $\Lambda$ is an antisymmetric $(2,0)$-tensor, $E$ is a vector field, and the equalities

$$
[\Lambda, \Lambda]=2 E \wedge \Lambda, \quad[E, \Lambda]=0
$$

are satisfied. Here $[\cdot, \cdot]$ is the Schouten-Nijenhuis bracket.

For $u \in C^{\infty}(M)$ one defines a Hamiltonian vector field by $X_{u}=[\Lambda, u]+$ $u E$. The orbits of the set of all Hamiltonian vector fields form a characteristic foliation of $(\Lambda, E)$, denoted by $\mathcal{F}(\Lambda, E)$. Next, let $\mathcal{F}^{*}(\Lambda, E)$ be the foliation determined by all $X_{u}$ with $u \in C^{\infty}(M)$ satisfying $\mathcal{L}_{E} u=0$. Then

$$
\mathcal{F}(E) \prec \mathcal{F}^{*}(\Lambda, E) \prec \mathcal{F}(\Lambda, E),
$$

where $\mathcal{F}(E)$ is the foliation given by the orbits of $E$ (cf. [3, p. 119]).

3.11. Interesting examples arise in multisymplectic geometry (see, e.g., $[4,5,7])$. One of them is produced by Nambu-Poisson manifolds.

Let us recall that a skew-symmetric $n$-linear mapping $\{, \ldots\}:, C^{\infty}(M) \times$ $\ldots \times C^{\infty}(M) \rightarrow C^{\infty}(M)$ is called a generalized almost Poisson (g.a.P.) 
bracket of order $n$ if it satisfies the Leibniz rule:

$$
\left\{u_{1} v_{1}, \ldots, u_{n}\right\}=u_{1}\left\{v_{1}, \ldots, u_{n}\right\}+v_{1}\left\{u_{1}, \ldots, u_{n}\right\}
$$

for all $u_{1}, \ldots, u_{n}, v_{1} \in C^{\infty}(M)$. Equivalently, a g.a.P. manifold of order $n$ is a pair $(M, \Lambda)$, where $\Lambda$ is a skew-symmetric $(n, 0)$-tensor on $M$. The relation between $\Lambda$ and the $n$-bracket $\{, \ldots$,$\} is expressed by the equal-$ ity $\Lambda\left(d u_{1}, \ldots, d u_{n}\right)=\left\{u_{1}, \ldots, u_{n}\right\}$. Then we define a linear mapping $\sharp$ : $\Omega^{n-1}(M) \rightarrow \mathcal{X}(M)$ by setting

$$
\left\langle\sharp\left(\alpha_{1} \wedge \ldots \wedge \alpha_{n-1}\right), \beta\right\rangle=\Lambda\left(\alpha_{1}, \ldots, \alpha_{n-1}, \beta\right)
$$

for any $\alpha_{1}, \ldots, \alpha_{n-1}, \beta \in \Omega^{1}(M)$. Here $\langle$,$\rangle is the natural pairing on \mathcal{X}(M) \times$ $\Omega^{1}(M)$.

For any $u_{1}, \ldots, u_{n-1} \in C^{\infty}(M)$ we define a Hamiltonian vector field

$$
X_{u_{1}, \ldots, u_{n-1}}=\sharp\left(d u_{1} \wedge \ldots \wedge d u_{n-1}\right) .
$$

A vector field $X$ is called an infinitesimal automorphism of $(M, \Lambda)$ if $\mathcal{L}_{X} \Lambda=$ 0 . It is visible that this condition amounts to claiming that $X$ is a derivation of the bracket $\{, \ldots$,$\} .$

Now a g.a.P. manifold of order $n$ is called a Nambu-Poisson manifold if any Hamiltonian vector field is a derivation of the bracket. Notice that for $n=2$ the above condition is equivalent to $[\Lambda, \Lambda]=0$ and, consequently, the Nambu-Poisson manifolds of order 2 coincide with the Poisson manifolds.

Given a Nambu-Poisson tensor let us define a smooth distribution $\mathcal{D}=$ $\left\{D_{x}\right\}_{x \in M}$ where $D_{x}=\sharp\left(\bigwedge^{n-1} T_{x}^{*} M\right)$. This distribution is called characteristic. We have the following structural theorem.

Theorem $3[4]$. Let $(M, \Lambda)$ be an m-dimensional Nambu-Poisson manifold of order $n \geq 3$. Then:

(1) The characteristic distribution $\mathcal{D}$ is completely integrable and, consequently, it defines a foliation, denoted by $\mathcal{F}(\Lambda)$. There are two kinds of leaves of $\mathcal{F}(\Lambda)$ : (a) if $\Lambda_{x}=0$ then the leaf passing through $x$ reduces to $x$ itself, and (b) if $\Lambda_{x} \neq 0$ then the leaf meeting $x$ has dimension $n$ and $\Lambda$ restricted to it induces a Nambu-Poisson structure which comes from a volume form.

(2) In case (b) there exists a distinguished chart $\left(x_{1}, \ldots, x_{n}, y_{1}, \ldots, y_{q}\right)$ at $x(q=m-n)$ such that $\Lambda=\partial_{1} \wedge, \ldots \wedge \partial_{n}$ where $\partial_{i}=\partial / \partial x_{i}$.

Now for any $u \in C^{\infty}(M)$ we put $\Lambda_{u}=\iota_{d u} \Lambda$. It is easily seen [4] that $\Lambda_{u}$ is a g.a.P. (resp. Nambu-Poisson) structure of order $n-1$ if $\Lambda$ is a g.a.P.(resp. Nambu-Poisson) structure of order $n$. This can be iterated by setting $\Lambda_{u v}=$ $\left(\Lambda_{u}\right)_{v}$ and so on. Therefore for any choice $u_{1}, \ldots, u_{n-1} \in C^{\infty}(M)$ we obtain 
a flag structure

$$
\mathcal{F}\left(\Lambda_{u_{1}, \ldots, u_{n-1}}\right) \prec \mathcal{F}\left(\Lambda_{u_{1}, \ldots, u_{n-2}}\right) \prec \ldots \prec \mathcal{F}\left(\Lambda_{u_{1}}\right) \prec \mathcal{F}(\Lambda) .
$$

As in (3.6) there are no common canonical charts for this flag.

3.12. Only recently the authors of [5] have introduced the concept of Nambu-Jacobi structure as a counterpart of the Jacobi structures in multisymplectic geometry. Specifically, if $\left(\Lambda_{1}, \Lambda_{2}\right)$ is a Nambu-Jacobi structure of order $n, n>2$, then $\Lambda_{1}$ (resp. $\Lambda_{2}$ ) is a Nambu-Poisson structure of order $n$ (resp. $n-1)$. Consequently, further examples of flag structures occur.

4. Locality and pseudo- $n$-transitivity. In [12] we have proven that the locality of a diffeomorphism group yields its pseudo- $n$-transitivity. This can be extended to the flag case.

Recall that $G(M) \subset \operatorname{Diff}^{r}(M)$, where $r \leq \infty$ (resp. $r=\omega$ ) satisfies the locality condition if for any open relatively compact $U, V \subset M$ with $\bar{U} \subset V$, and a $C^{r}$-diffeotopy $\left\{f_{t}\right\}$ in $G(M)$ with $f_{0}=\mathrm{id}$, there exist $\varepsilon>0$ and a smooth diffeotopy $\left\{g_{t}\right\}$ in $G(M)$ such that $g_{t}=f_{t}$ on $U$ and $\operatorname{supp}\left(g_{t}\right) \subset V$ for $|t|<\varepsilon$ (resp. $g_{t}$ is sufficiently $C^{1}$ near $f_{t}$ on $U$ and $g_{t}$ is sufficiently $C^{1}$ near the identity outside $V$ for $|t|<\varepsilon$ ).

By an orbit of a sequence of diffeomorphism groups $G_{1}(M) \subset \ldots \subset$ $G_{k}(M)$ we mean any orbit of each $G_{i}(M)_{0}$. Next, for $x, y \in M$ belonging to a common orbit, a minimal orbit containing $x, y$ is the unique orbit of the least dimension passing through $x, y$.

The sequence $G_{1}(M) \subset \ldots \subset G_{k}(M)$ is said to be pseudo- $n$-transitive if for any two $n$-tuples of pairwise distinct points $\left(x_{1}, \ldots, x_{n}\right)$ and $\left(y_{1}, \ldots, y_{n}\right)$ of $M$ such that $x_{j}, y_{j}$ belong to the same orbit and each orbit of dimension $\leq 1$ contains at most one $x_{j}$ there exists $f \in G_{k}(M)_{0}$ satisfying $f\left(x_{j}\right)=y_{j}$ and preserving all the minimal orbits containing the pairs $x_{j}, y_{j}$.

Observe that the concept of pseudo- $n$-transitivity is an extension of the well-known notion of $n$-transitivity (see, e.g., [8]) to arbitrary groups of diffeomorphisms.

THEOREM 4. Suppose that each group of an increasing sequence $G_{1}(M) \subset$ $\ldots \subset G_{k}(M) \subset \operatorname{Diff}^{r}(M)(1 \leq r \leq \omega)$ satisfies the locality condition. Then this sequence is pseudo-n-transitive for every $n \geq 1$.

The proof makes use of our Lemma and repeats an argument from [12].

\section{References}

[1] M. Bauer, Feuilletage singulier défini par une distribution presque régulière, Thèse, Univ. Louis Pasteur (Strasbourg), Publ. I.R.M.A., 1985.

[2] P. Dazord, Feuilletages à singularités, Indag. Math. 47 (1985), 21-39. 
[3] P. Dazord, A. Lichnerowicz et C. M. Marle, Structure locale des variétés de Jacobi, J. Math. Pures Appl. 70 (1991), 101-152.

[4] R. Ibáñez, M. de León, J. C. Marrero and D. Martin de Diego, Dynamics of generalized Poisson and Nambu-Poisson brackets, J. Math. Phys. 38 (1997), $2332-2344$.

[5] R. Ibáñez, M. de León, J. C. Marrero and E. Padrón, Nambu-Jacobi and generalized Jacobi manifolds, preprint, 1997.

[6] C. M. Marle, Lie group actions on a canonical manifold, in: Symplectic Geometry, A. Crumeyrolle and J. Grifone (eds.), Pitman, Boston, 1983, 144-166.

[7] P. W. Michor and A. M. Vinogradov, $n$-ary Lie and associative algebras, preprint ESI 402, 1996.

[8] P. W. Michor and C. Vizman, n-transitivity of certain diffeomorphism groups, Acta Math. Univ. Comenian. 63 (1994), 221-225.

[9] P. Molino, Riemannian Foliations, Progr. Math. 73, Birkhäuser, 1988.

[10] -, Orbit-like foliations, in: Geometric Study of Foliations (Tokyo, 1993), World Sci., Singapore, 1994, 97-119.

[11] R. Ouzilou, Hamiltonian actions on Poisson manifolds, in: Symplectic Geometry, A. Crumeyrolle and J. Grifone (eds.), Pitman, Boston, 1983, 172-183.

[12] T. Rybicki, Pseudo-n-transitivity of the automorphism group of a geometric structure, Geom. Dedicata 67 (1997), 181-186.

[13] P. Stefan, Accessibility and foliations with singularities, Bull. Amer. Math. Soc. 80 (1974), 1142-1145.

[14] - Accessible sets, orbits and foliations with singularities, Proc. London Math. Soc. 29 (1974), 699-713.

[15] H. J. Sussmann, Orbits of families of vector fields and integrability of distributions, Trans. Amer. Math. Soc. 180 (1973), 171-188.

[16] I. Vaisman, Lectures on the Geometry of Poisson Manifolds, Progr. Math. 118, Birkhäuser, Basel, 1994.

[17] V. P. Vilflyantsev, Frobenius theorem for differential systems with singularities, Vestnik Moskov. Univ. 3 (1980), 11-14 (in Russian).

[18] R. A. Wolak, Characteristic classes of almost-flag structures, Geom. Dedicata 24 (1987), 207-220.

Institute of Mathematics

Pedagogical University

Rejtana $16 \mathrm{~A}$

35-310 Rzeszów, Poland

E-mail: rybicki@im.uj.edu.pl 